\title{
QUATERNIONIC KÄHLER MANIFOLDS WITH HERMITIAN AND NORDEN METRICS
}

\author{
MANCHO MANEV
}

\begin{abstract}
Almost hypercomplex manifolds with Hermitian and Norden metrics and more specially the corresponding quaternionic Kähler manifolds are considered. Some necessary and sufficient conditions the investigated manifolds be isotropic hyper-Kählerian and flat are found. It is proved that the quaternionic Kähler manifolds with the considered metric structure are Einstein for dimension at least 8 . The class of the non-hyper-Kähler quaternionic Kähler manifold of the considered type is determined.
\end{abstract}

\section{Contents}

Introduction

1. Almost hypercomplex manifolds with NH-metric structure

2. Quaternionic Kähler manifolds with NH-metric structure

3. Quaternionic Kähler NH-manifolds in a classification of almost hypercomplex NH-manifolds

4. Non-hyper-Kähler quaternionic Kähler NH-manifolds

References

\section{INTRODUCTION}

In this work we continue the investigations on a manifold $M$ with an almost hypercomplex structure $H$. We equip this almost hypercomplex manifold $(M, H)$ with a metric structure $G$, generated by a pseudo-Riemannian metric $g$ of neutral signature $([4,[5])$.

It is known, if $g$ is a Hermitian metric on $(M, H)$, the derived metric structure $G$ is the known hyper-Hermitian structure. It consists of the given Hermitian metric $g$ with respect to the three almost complex structures of $H$ and the three Kähler forms associated with $g$ by $H$ [1].

In our case the considered metric structure $G$ has a different type of compatibility with $H$. The structure $G$ is generated by a neutral metric $g$ such that the one (resp., the other two) of the almost complex structures of $H$ acts as an isometry (resp., act as anti-isometries) with respect to $g$ in each tangent fibre. Let the almost complex structures of $H$ act as isometries or anti-isometries with respect to the metric. Then

2000 Mathematics Subject Classification. 53C26, 53C15, 53C50, 53C55.

Key words and phrases. almost hypercomplex manifold, quaternionic Kähler manifold, Norden metric, indefinite metric.

${ }^{*}$ This work was partially supported by the Scientific Researches Fund at the University of Plovdiv. 
the existence of an anti-isometry generates exactly the existence of one more antiisometry and an isometry. Thus, $G$ contains the metric $g$ and three $(0,2)$-tensors associated by $H$ - a Kähler form and two metrics of the same type. The existence of such bilinear forms on an almost hypercomplex manifold is proved in [4. The neutral metric $g$ is Hermitian with respect to the one almost complex structure of $H$ and $g$ is an anti-Hermitian (i. e. a Norden) metric regarding the other two almost complex structures of $H$. For this reason we call the derived almost hypercomplex manifold $(M, H, G)$ an almost hypercomplex manifold with $\mathrm{NH}$-metric structure or an almost hypercomplex NH-manifold.

If the three almost complex structures of $H$ are parallel with respect to the LeviCivita connection $\nabla$ of $g$ then such hypercomplex NH-manifolds of Kähler type we call hyper-Kähler $\mathrm{NH}$-manifolds, which are flat according to [5].

In the first section we recall some facts about the almost hypercomplex NHmanifolds known from [1, 4], [5, 7].

In the second section we introduce the corresponding quaternionic Kähler manifold of an almost hypercomplex manifold with NH-metric structure. We establish that the quaternionic Kähler NH-manifolds are Einstein for dimension $4 n \geq 8$. For comparison, it is known that the quaternionic Kähler manifolds with hyperHermitian metric structure are Einstein for all dimensions.

In the third section we consider the location of the quaternionic Kähler NHmanifolds in the classification of the corresponding almost hypercomplex manifolds with respect to the covariant derivatives of the almost complex structures. We get only one class (except the general one) of the considered classification where these manifolds are non-hyper-Kählerian and consequently non-flat always.

In the fourth section we characterize the obtained in the previous chapter nonhyper-Kähler quaternionic Kähler NH-manifolds.

The basic problem of this work is the existence and the geometric characteristics of the quaternionic Kähler NH-manifolds. The main results of this paper is that every quaternionic Kähler NH-manifold is Einstein for dimension at least 8 and it is not flat hyper-Kählerian only when belongs to the general class $\mathcal{W}_{1} \oplus \mathcal{W}_{2} \oplus \mathcal{W}_{3}$ or the class $\mathcal{W}_{1} \oplus \mathcal{W}_{3}$, where the manifold is Ricci-symmetric.

\section{Almost hypercomplex manifolds with NH-Metric Structure}

Let $(M, H)$ be an almost hypercomplex manifold, i. e. $M$ is a $4 n$-dimensional differentiable manifold and $H=\left(J_{1}, J_{2}, J_{3}\right)$ is a triple of almost complex structures on $M$ with the properties:

$$
J_{\alpha}=J_{\beta} \circ J_{\gamma}=-J_{\gamma} \circ J_{\beta}, \quad J_{\alpha}^{2}=-I
$$

for all cyclic permutations $(\alpha, \beta, \gamma)$ of $(1,2,3)$ and $I$ denotes the identity [1].

Let $g$ be a neutral metric on $(M, H)$ with the properties

$$
g(x, y)=\varepsilon_{\alpha} g\left(J_{\alpha} x, J_{\alpha} y\right)
$$

where

$$
\varepsilon_{\alpha}= \begin{cases}1, & \alpha=1 \\ -1, & \alpha=2 ; 3 .\end{cases}
$$

Moreover, the associated (Kähler) 2 -form $g_{1}$ and the associated neutral metrics $g_{2}$ and $g_{3}$ are determined by

$$
g_{\alpha}(x, y)=g\left(J_{\alpha} x, y\right)=-\varepsilon_{\alpha} g\left(x, J_{\alpha} y\right) .
$$


A structure $(H, G)=\left(J_{1}, J_{2}, J_{3}, g, g_{1}, g_{2}, g_{3}\right)$ is introduced and investigated in [4], [5], 8], 6] and [7. Now we call it an almost hypercomplex NH-metric structure on $M$. Then, a manifold with such structure $(M, H, G)$ we call an almost hypercomplex manifold with $\mathrm{NH}$-metric structure or an almost hypercomplex NH-manifold.

The structural tensors of a such manifold are the following three $(0,3)$-tensors

$$
F_{\alpha}(x, y, z)=g\left(\left(\nabla_{x} J_{\alpha}\right) y, z\right)=\left(\nabla_{x} g_{\alpha}\right)(y, z),
$$

where $\nabla$ is the Levi-Civita connection generated by $g$. The corresponding Lie 1forms $\theta_{\alpha}$ are defined by

$$
\theta_{\alpha}(\cdot)=g^{i j} F_{\alpha}\left(e_{i}, e_{j}, \cdot\right)
$$

for an arbitrary basis $\left\{e_{1}, e_{2}, \ldots, e_{4 n}\right\}$ of $T_{p} M, p \in M$.

In [5] we study the so-called hyper-Kähler manifolds with NH-metric structure (or pseudo-hyper-Kähler manifolds), i. e. the almost hypercomplex NH-manifold in the class $\mathcal{K}$, where $\nabla J_{\alpha}=0$ for all $\alpha=1,2,3$. A sufficient condition $(M, H, G)$ be in $\mathcal{K}$ is this manifold be of Kähler-type with respect to two of the three complex structures of $H$ [4].

As $g$ is an indefinite metric, there exist isotropic vectors $x$ on $M$, i. e. $g(x, x)=0$, $x \neq 0$. In [4] we define the invariant square norm

$$
\left\|\nabla J_{\alpha}\right\|^{2}=g^{i j} g^{k l} g\left(\left(\nabla_{i} J_{\alpha}\right) e_{k},\left(\nabla_{j} J_{\alpha}\right) e_{l}\right),
$$

where $\left\{e_{1}, e_{2}, \ldots, e_{4 n}\right\}$ of $T_{p} M, p \in M$. We say that an almost hypercomplex NHmanifold is an isotropic hyper-Kähler NH-manifold if $\left\|\nabla J_{\alpha}\right\|^{2}=0$ for every $J_{\alpha}$ of $H$. In 4 such a manifold is called an isotropic hyper-Kähler manifold. Clearly, if $(M, H, G)$ is a hyper-Kähler NH-manifold, then it is an isotropic hyper-Kähler $\mathrm{NH}$-manifold. The inverse statement does not hold.

Let us consider the Nijenhuis tensors $N_{\alpha}$ for $J_{\alpha}$ given by $N_{\alpha}(x, y)=\left[J_{\alpha} x, J_{\alpha} y\right]-$ $J_{\alpha}\left[J_{\alpha} x, y\right]-J_{\alpha}\left[x, J_{\alpha} y\right]-[x, y]$ for $x, y \in T_{p} M$. It is well known that the almost hypercomplex structure $H=\left(J_{\alpha}\right)$ is a hypercomplex structure if $N_{\alpha}$ vanishes for each $\alpha=1,2,3$. Moreover, it is known that one almost hypercomplex structure $H$ is hypercomplex if and only if two of the structures $J_{\alpha}(\alpha=1,2,3)$ are integrable. This means that two of the tensors $N_{\alpha}$ vanish [1.

Let us note that according to (1.2) the manifold $\left(M, J_{1}, g\right)$ is almost Hermitian and the manifolds $\left(M, J_{\alpha}, g\right), \alpha=2,3$, are almost complex manifolds with NHmetric structure (or $B$-metric) [2. The basic classes of the mentioned two types of manifolds are given in 3 and 2, respectively, and they are determined for dimension $4 n$ as follows:

$$
\begin{aligned}
& \mathcal{W}_{1}\left(J_{1}\right): F_{1}(x, y, z)=-F_{1}(y, x, z) ; \\
& \mathcal{W}_{2}\left(J_{1}\right): \underset{s, y, z}{\mathfrak{S}\left\{F_{1}(x, y, z)\right\}=0 ;} \\
& \mathcal{W}_{3}\left(J_{1}\right): F_{1}(x, y, z)=F_{1}\left(J_{1} x, J_{1} y, z\right), \quad \theta_{1}=0 ; \\
& \mathcal{W}_{4}\left(J_{1}\right): F_{1}(x, y, z)=\frac{1}{4 n-2}\left\{g(x, y) \theta_{1}(z)-g(x, z) \theta_{1}(y)\right. \\
& \left.\quad-g\left(x, J_{1} y\right) \theta_{1}\left(J_{1} z\right)+g\left(x, J_{1} z\right) \theta_{1}\left(J_{1} y\right)\right\}
\end{aligned}
$$


and for $\alpha=2$ or 3

$$
\begin{aligned}
& \mathcal{W}_{1}\left(J_{\alpha}\right): F_{\alpha}(x, y, z)=\frac{1}{4 n}\{ \\
&(x, y) \theta_{\alpha}(z)+g(x, z) \theta_{\alpha}(y) \\
&\left.+g\left(x, J_{\alpha} y\right) \theta_{\alpha}\left(J_{\alpha} z\right)+g\left(x, J_{\alpha} z\right) \theta_{\alpha}\left(J_{\alpha} y\right)\right\} ; \\
& \mathcal{W}_{2}\left(J_{\alpha}\right): \underset{x, y, z}{\mathfrak{S}}\left\{F_{\alpha}\left(x, y, J_{\alpha} z\right)\right\}=0, \quad \theta_{\alpha}=0 \\
& \mathcal{W}_{3}\left(J_{\alpha}\right): \underset{x, y, z}{\mathfrak{S}}\left\{F_{\alpha}(x, y, z)\right\}=0,
\end{aligned}
$$

where $\mathfrak{S}$ is the cyclic sum by three arguments $x, y, z$.

The special class $\mathcal{W}_{0}\left(J_{\alpha}\right): F_{\alpha}=0(\alpha=1,2,3)$ of the Kähler-type manifolds belongs to any other class within the corresponding classification.

Let the curvature tensor $R$ of the Levi-Civita connection $\nabla$, generated by $g$, be defined, as usually, by $R(x, y) z=\left[\nabla_{x}, \nabla_{y}\right] z-\nabla_{[x, y]} z$. The corresponding $(0,4)$ tensor is determined by $R(x, y, z, w)=g(R(x, y) z, w)$. Obviously, $R$ is a Kähler-type tensor on an arbitrary hyper-Kähler NH-manifold, i. e.

$$
R(x, y, z, w)=\varepsilon_{\alpha} R\left(x, y, J_{\alpha} z, J_{\alpha} w\right)=\varepsilon_{\alpha} R\left(J_{\alpha} x, J_{\alpha} y, z, w\right) .
$$

A basic property of the hyper-Kähler NH-manifolds is given in [5] by the following

Theorem 1.1 ([5]). Each hyper-Kähler NH-manifold is a flat pseudo-Riemannian manifold of signature $(2 n, 2 n)$.

In 7 it is proved the following more general property.

Theorem 1.2 ([7]). Every Kähler-type tensor on an almost hypercomplex NH-manifold is zero.

\section{Quaternionic KÄHLER MANifolds With NH-Metric Structure}

Let us consider again only an almost hypercomplex manifold $(M, H)$. The endomorphism $Q=\lambda_{1} J_{1}+\lambda_{2} J_{2}+\lambda_{3} J_{3}, \lambda_{i} \in \mathbb{R}$, is called a quaternionic structure on $(M, H)$ with an admissible basis $H$. A quaternionic structure with the condition $\nabla Q=0$ is called a quaternionic Kähler structure on $(M, H)$. An almost hypercomplex manifold with quaternionic Kähler structure is determined by

$$
\left(\nabla_{x} J_{\alpha}\right) y=\omega_{\gamma}(x) J_{\beta} y-\omega_{\beta}(x) J_{\gamma} y
$$

for all cyclic permutations $(\alpha, \beta, \gamma)$ of $(1,2,3)$, where $\omega_{\alpha}$ are local 1-forms associated to $H=\left(J_{\alpha}\right), \alpha=1,2,3$. [1]

Next, we equip the quaternionic Kähler manifold with an NH-metric structure $G=\left(g, g_{1}, g_{2}, g_{3}\right)$, determined by (1.2) and (1.3), and obtain a quaternionic Kähler manifold with $\mathrm{NH}$-metric structure or a quaternionic Kähler NH-manifold.

Having in mind (2.1) and (1.6), for a quaternionic Kähler NH-manifold we obtain the following form of the square norm of $\nabla J_{\alpha}$ :

$$
\left\|\nabla J_{\alpha}\right\|^{2}=4 n\left\{\varepsilon_{\beta} \omega_{\gamma}\left(\Omega_{\gamma}\right)+\varepsilon_{\gamma} \omega_{\beta}\left(\Omega_{\beta}\right)\right\},
$$

where $\Omega_{1}, \Omega_{2}, \Omega_{3}$ are the corresponding vectors of $\omega_{1}, \omega_{2}, \omega_{3}$ regarding $g$, respectively. Therefore we have immediately the following

Proposition 2.1. A quaternionic Kähler NH-manifold is an isotropic hyper-Kähler $N H$-manifold iff the corresponding vectors of the 1-forms $\omega_{1}, \omega_{2}$ and $\omega_{3}$ with respect to $g$ are isotropic vectors regarding $g$. 
Having in mind (2.1), we obtain the following property for all cyclic permutations $(\alpha, \beta, \gamma)$ of $(1,2,3)$ :

$$
R(x, y) J_{\alpha} z=J_{\alpha} R(x, y) z-\eta_{\beta}(x, y) J_{\gamma} z+\eta_{\gamma}(x, y) J_{\beta} z
$$

where

$$
\eta_{\beta}(x, y)=\mathrm{d} \omega_{\beta}(x, y)+\omega_{\gamma}(x) \omega_{\alpha}(y)-\omega_{\alpha}(x) \omega_{\gamma}(y)
$$

are 2 -forms associated to the local 1-forms $\omega_{1}, \omega_{2}, \omega_{3}$ and therefore

$$
R(x, y, z, w)-\varepsilon_{\alpha} R\left(x, y, J_{\alpha} z, J_{\alpha} w\right)=\eta_{\beta}(x, y) g_{\beta}(z, w)+\eta_{\gamma}(x, y) g_{\gamma}(z, w) .
$$

According to the antisymmetry of $R$ by the third and the forth entries, we establish that $\eta_{2}=\eta_{3}=0$, i. e.

Lemma 2.2. The local 1-forms $\omega_{1}, \omega_{2}$ and $\omega_{3}$, determining a quaternionic Kähler NH-manifold, satisfy the following identities

$$
\begin{aligned}
& \mathrm{d} \omega_{2}(x, y)=-\omega_{3}(x) \omega_{1}(y)+\omega_{1}(x) \omega_{3}(y), \\
& \mathrm{d} \omega_{3}(x, y)=-\omega_{1}(x) \omega_{2}(y)+\omega_{2}(x) \omega_{1}(y) .
\end{aligned}
$$

Then, according to (2.6), equations (2.5) take the form

$$
\begin{gathered}
R\left(x, y, J_{1} z, J_{1} w\right)=R(x, y, z, w), \\
R\left(x, y, J_{2} z, J_{2} w\right)=R\left(x, y, J_{3} z, J_{3} w\right)=-R(x, y, z, w)+\eta_{1}(x, y) g_{1}(z, w) .
\end{gathered}
$$

Having in mind (2.7), (2.8) and (1.9), we have immediately

Lemma 2.3. The curvature tensor $R$ of a quaternionic Kähler NH-manifold is of Kähler-type iff $\eta_{1}=0, i$. e. the following condition is valid

$$
\mathrm{d} \omega_{1}(x, y)=-\omega_{2}(x) \omega_{3}(y)+\omega_{3}(x) \omega_{2}(y) .
$$

According to Lemma 2.3 and Theorem 1.1, we have

Proposition 2.4. The necessary and sufficient condition an arbitrary quaternionic Kähler NH-manifold be flat is condition (2.9).

Lemma 2.5. The Ricci tensor and the 2-form $\eta_{1}$, defined by (2.4), have the following relation on any quaternionic Kähler NH-manifold:

$$
\rho(x, y)=n \eta_{1}\left(J_{1} x, y\right) .
$$

Proof. From (2.8) for $z \rightarrow e_{i}, w \rightarrow J_{1} e_{j}$ by contraction with $g^{i j}$ we have

$$
\begin{aligned}
-g^{i j} R\left(x, y, J_{2} e_{i}, J_{3} e_{j}\right) & =g^{i j} R\left(x, y, J_{3} e_{i}, J_{2} e_{j}\right) \\
& =-g^{i j} R\left(x, y, e_{i}, J_{1} e_{j}\right)+4 n \eta_{1}(x, y) .
\end{aligned}
$$

Having in mind the antisymmetry on the second pair arguments of $R$ and $J_{1}=J_{2} J_{3}$, we get

$$
-g^{i j} R\left(x, y, J_{2} e_{i}, J_{3} e_{j}\right)=g^{i j} R\left(x, y, J_{3} e_{i}, J_{2} e_{j}\right)=g^{i j} R\left(x, y, e_{i}, J_{1} e_{j}\right) .
$$

and therefore from (2.11) we have

$$
g^{i j} R\left(x, y, e_{i}, J_{1} e_{j}\right)=2 n \eta_{1}(x, y) .
$$


After that, from (2.12), applying the properties of the curvature tensor $R$, (1.2) for $\alpha=1$ and (2.7), we obtain consequently

$$
\begin{aligned}
& 2 n \eta_{1}(x, y)=g^{i j} R\left(x, y, e_{i}, J_{1} e_{j}\right)=g^{i j}\left\{-R\left(x, e_{i}, J_{1} e_{j}, y\right)-R\left(x, J_{1} e_{j}, y, e_{i}\right)\right\} \\
& =g^{i j} R\left(x, e_{i}, y, J_{1} e_{j}\right)+g^{i j} R\left(x, e_{j}, y, J_{1} e_{i}\right)=2 g^{i j} R\left(x, e_{i}, y, J_{1} e_{j}\right) \\
& =-2 g^{i j} R\left(e_{i}, x, y, J_{1} e_{j}\right)=2 g^{i j} R\left(e_{i}, x, J_{1} y, e_{j}\right)=2 \rho\left(x, J_{1} y\right),
\end{aligned}
$$

i. e.

$$
\eta_{1}(x, y)=\frac{1}{n} \rho\left(x, J_{1} y\right) .
$$

Because of the symmetry of $\rho$ and the antisymmetry of $\eta_{1}$ we have the property

$$
\eta_{1}\left(x, J_{1} y\right)=-\eta_{1}\left(J_{1} x, y\right)
$$

and therefore

$$
\rho\left(J_{1} x, J_{1} y\right)=\rho(x, y) .
$$

Hence, from (2.13), (2.14) and (2.15), we obtain (2.10).

Proposition 2.6. A quaternionic Kähler NH-manifold is Ricci-flat iff it is flat.

Proof. Using Lemma 2.5] property (2.8) takes the form

$$
\begin{aligned}
R\left(x, y, J_{2} z, J_{2} w\right) & =R\left(x, y, J_{3} z, J_{3} w\right) \\
& =-R(x, y, z, w)-\frac{1}{n} \rho\left(J_{1} x, y\right) g\left(J_{1} z, w\right) .
\end{aligned}
$$

Then, according to (2.16), (1.9) and Theorem 1.2, we obtain the equivalence in the statement.

Theorem 2.7. Quaternionic Kähler manifolds with NH-metric structure are Einstein for dimension $4 n \geq 8$.

Proof. By virtue of (2.7), (2.16) and (2.15) we obtain the following properties

$$
\begin{gathered}
R\left(J_{1} x, J_{1} y, J_{1} z, J_{1} w\right)=R(x, y, z, w), \\
R\left(J_{2} x, J_{2} y, J_{2} z, J_{2} w\right)=R\left(J_{3} x, J_{3} y, J_{3} z, J_{3} w\right) \\
=R(x, y, z, w)-\frac{1}{n} g\left(x, J_{1} y\right) \rho\left(J_{1} z, w\right)+\frac{1}{n} \rho\left(J_{2} x, J_{3} y\right) g\left(J_{1} z, w\right) .
\end{gathered}
$$

Hence, for the Ricci tensor we have (2.15) and

$$
\left(n^{2}-1\right) \rho\left(J_{2} y, J_{2} z\right)=\left(n^{2}-1\right) \rho\left(J_{3} y, J_{3} z\right)=-\left(n^{2}-1\right) \rho(y, z) .
$$

Then for $n>1$ the Ricci tensor is hybrid with respect to $J_{2}$ and $J_{3}$, i. e.

$$
\rho\left(J_{2} y, J_{2} z\right)=\rho\left(J_{3} y, J_{3} z\right)=-\rho(y, z) .
$$

The conditions (2.7), (2.8) and (2.10) imply for $n>1$ the following

$$
A(x, z)=-\frac{2}{n} \rho(x, x) g(z, z)=-\frac{2}{n} g(x, x) \rho(z, z),
$$

where

$$
\begin{aligned}
A(x, z)= & R\left(x, J_{1} x, z, J_{1} z\right)-R\left(x, J_{1} x, J_{2} z, J_{3} z\right) \\
& -R\left(J_{2} x, J_{3} x, z, J_{1} z\right)+R\left(J_{2} x, J_{3} x, J_{2} z, J_{3} z\right)
\end{aligned}
$$

Then for arbitrary non-isotropic vectors we have $\rho=\lambda g, \lambda \in \mathbb{R}$. 
By Theorem 2.7 identity (2.16) implies the following corollary for $n \geq 2$ :

$$
\begin{aligned}
R\left(x, y, J_{2} z, J_{2} w\right) & =R\left(x, y, J_{3} z, J_{3} w\right) \\
& =-R(x, y, z, w)-\frac{\tau}{4 n^{2}} g\left(J_{1} x, y\right) g\left(J_{1} z, w\right) .
\end{aligned}
$$

Therefore from (2.20), using (1.9) and Theorem 1.2, we obtain the following

Proposition 2.8. A quaternionic Kähler NH-manifold of dimension $4 n \geq 8$ is scalar flat iff it is flat.

Proposition 2.9. A quaternionic Kähler NH-manifold of dimension $4 n \geq 8$ is determined by the local 1-forms satisfying the conditions (2.6) and

$$
\mathrm{d} \omega_{1}(x, y)=-\omega_{2}(x) \omega_{3}(y)+\omega_{3}(x) \omega_{2}(y)-\frac{\tau}{4 n^{2}} g\left(J_{1} x, y\right) .
$$

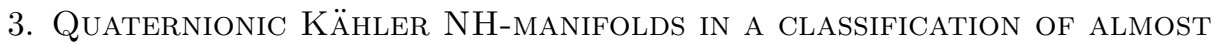 HYPERCOMPLEX NH-MANIFOLDS}

Firstly, let us consider the case when $H$ is (integrable) hypercomplex structure, i. e. when $N_{\alpha}$ vanishes for each $\alpha=1,2,3$.

The Nijenhuis tensor and its associated tensor for each $J_{\alpha}$ are determined as follows:

$$
\begin{aligned}
& N_{\alpha}(x, y)=\left(\nabla_{x} J_{\alpha}\right) J_{\alpha} y-\left(\nabla_{y} J_{\alpha}\right) J_{\alpha} x+\left(\nabla_{J_{\alpha} x} J_{\alpha}\right) y-\left(\nabla_{J_{\alpha} y} J_{\alpha}\right) x, \\
& N_{\alpha}^{*}(x, y)=\left(\nabla_{x} J_{\alpha}\right) J_{\alpha} y+\left(\nabla_{y} J_{\alpha}\right) J_{\alpha} x+\left(\nabla_{J_{\alpha} x} J_{\alpha}\right) y+\left(\nabla_{J_{\alpha} y} J_{\alpha}\right) x .
\end{aligned}
$$

Therefore, according to (2.1), for the quaternionic Kähler manifolds we have

$$
\begin{aligned}
N_{\alpha}(x, y)= & -\left[\omega_{\gamma}(x)+\omega_{\beta}\left(J_{\alpha} x\right)\right] J_{\gamma} y-\left[\omega_{\beta}(x)-\omega_{\gamma}\left(J_{\alpha} x\right)\right] J_{\beta} y \\
& +\left[\omega_{\gamma}(y)+\omega_{\beta}\left(J_{\alpha} y\right)\right] J_{\gamma} x+\left[\omega_{\beta}(y)-\omega_{\gamma}\left(J_{\alpha} y\right)\right] J_{\beta} x, \\
N_{\alpha}^{*}(x, y)= & -\left[\omega_{\gamma}(x)+\omega_{\beta}\left(J_{\alpha} x\right)\right] J_{\gamma} y-\left[\omega_{\beta}(x)-\omega_{\gamma}\left(J_{\alpha} x\right)\right] J_{\beta} y \\
& -\left[\omega_{\gamma}(y)+\omega_{\beta}\left(J_{\alpha} y\right)\right] J_{\gamma} x-\left[\omega_{\beta}(y)-\omega_{\gamma}\left(J_{\alpha} y\right)\right] J_{\beta} x .
\end{aligned}
$$

The last equations imply immediately the next two lemmas.

Lemma 3.1. The tensors $N_{\alpha}$ and $N_{\alpha}^{*}$ vanish iff $\omega_{\gamma}=-\omega_{\beta} \circ J_{\alpha}$ for any fixed cyclic permutation $(\alpha, \beta, \gamma)$ of $(1,2,3)$.

Lemma 3.2. The tensors $N_{\alpha}$ and $N_{\alpha}^{*}(\alpha=1,2,3)$ vanish iff

$$
\omega_{\alpha}=\omega_{\beta} \circ J_{\gamma}=-\omega_{\gamma} \circ J_{\beta}
$$

for cyclic permutations $(\alpha, \beta, \gamma)$ of $(1,2,3)$.

Now, according to (2.1) and (1.3), the structural tensors and their corresponding Lie 1-forms of the derived quaternionic Kähler NH-manifold, defined by (1.4) and (1.5), have the form

$$
\begin{gathered}
F_{\alpha}(x, y, z)=\omega_{\gamma}(x) g\left(J_{\beta} y, z\right)-\omega_{\beta}(x) g\left(J_{\gamma} y, z\right), \\
\theta_{\alpha}(z)=-\varepsilon_{\beta} \omega_{\gamma}\left(J_{\beta} z\right)+\varepsilon_{\gamma} \omega_{\beta}\left(J_{\gamma} z\right) .
\end{gathered}
$$


Proposition 3.3. If a quaternionic Kähler $N H$-manifold $(M, H, G)$ is integrable, then it is a hyper-Kähler NH-manifold, i. e.

$$
\begin{gathered}
(M, H, G) \in\left(\mathcal{W}_{3} \oplus \mathcal{W}_{4}\right)\left(J_{1}\right) \cap\left(\mathcal{W}_{1} \oplus \mathcal{W}_{2}\right)\left(J_{2}\right) \cap\left(\mathcal{W}_{1} \oplus \mathcal{W}_{2}\right)\left(J_{3}\right) \\
\Rightarrow \quad(M, H, G) \in \mathcal{K} .
\end{gathered}
$$

Proof. Let $(M, H, G)$ be an integrable hypercomplex NH-manifold, i. e. $(M, H, G)$ belongs to the class $\mathcal{W}_{3} \oplus \mathcal{W}_{4}$ with respect to $J_{1}$ in (1.7) and $(M, H, G)$ is an element of $\mathcal{W}_{1} \oplus \mathcal{W}_{2}$ regarding $J_{2}$ and $J_{3}$, according to (1.8).

Therefore $N_{1}=N_{2}=N_{3}=0$ hold and then, according to Lemma 3.2, conditions (3.3) are valid. Hence, according to $\varepsilon_{\alpha}+\varepsilon_{\beta}+\varepsilon_{\gamma}=-1$ and $\varepsilon_{\alpha} \varepsilon_{\beta} \varepsilon_{\gamma}=1$, relation (3.5) takes the form

which imply

$$
\theta_{\alpha}=-\left(1+\varepsilon_{\alpha}\right) \omega_{\alpha}
$$

$$
\theta_{1}=-2 \omega_{1}, \quad \theta_{2}=\theta_{3}=0 .
$$

On the other hand, $N_{\alpha}=N_{\alpha}^{*}=0$ and (3.1) imply $\left(\nabla_{x} J_{\alpha}\right) y=\left(\nabla_{J_{\alpha} x} J_{\alpha}\right) J_{\alpha} y$ and finally the fact that the manifold is hyper-Kählerian with an NH-metric structure.

Proposition 3.4. If an almost hypercomplex $N H$-manifold $(M, H, G)$, determined by the properties $\theta_{2}=\theta_{3}=0$, is quaternionic Kählerian, then it is a hyper-Kähler NH-manifold, i. e.

$$
(M, H, G) \in\left(\mathcal{W}_{2} \oplus \mathcal{W}_{3}\right)\left(J_{2}\right) \cap\left(\mathcal{W}_{2} \oplus \mathcal{W}_{3}\right)\left(J_{3}\right) \quad \Rightarrow \quad(M, H, G) \in \mathcal{K} .
$$

Proof. Since $\theta_{2}=\theta_{3}=0$, we have $N_{2}=N_{3}=0$, because of (3.5) and (3.2). Consequently, $N_{1}$ vanishes, too. Then, according to Proposition 3.3 and conditions (1.8), the considered manifold belongs to the class $\mathcal{K}$.

Let us remark, using (1.8), that an almost complex manifold with Norden metric belongs to $\mathcal{W}_{1} \oplus \mathcal{W}_{3}$ regarding $J_{\alpha}$ iff the following property holds for $\alpha=2$ or 3 , respectively

$$
\underset{x, y, z}{\mathfrak{S}} F_{\alpha}(x, y, z)=\frac{1}{2 n} \underset{x, y, z}{\mathfrak{S}}\left\{g(x, y) \theta_{\alpha}(z)+g\left(J_{\alpha} x, y\right) \theta_{\alpha}\left(J_{\alpha} z\right)\right\} .
$$

Proposition 3.5. Let $(M, H, G)$ be an almost hypercomplex $N H$-manifold belonging to the class $\mathcal{W}_{1} \oplus \mathcal{W}_{3}$ with respect to $J_{2}$ and $J_{3}$. If $(M, H, G)$ is quaternionic Kählerian, then it is a Kähler manifold with respect to $J_{1}$, i. e.

$$
(M, H, G) \in\left(\mathcal{W}_{1} \oplus \mathcal{W}_{3}\right)\left(J_{2}\right) \cap\left(\mathcal{W}_{1} \oplus \mathcal{W}_{3}\right)\left(J_{3}\right) \quad \Rightarrow \quad(M, H, G) \in \mathcal{W}_{0}\left(J_{1}\right) .
$$

Moreover, we have

$$
\begin{array}{lll}
\left(\nabla_{x} J_{1}\right) y=0, & \\
\left(\nabla_{x} J_{2}\right) y=\omega_{1}(x) J_{3} y, & \omega_{1}(x)=-\theta_{2}\left(J_{3} x\right), \\
\left(\nabla_{x} J_{3}\right) y=-\omega_{1}(x) J_{2} y, & \omega_{1}(x)=\theta_{3}\left(J_{2} x\right) .
\end{array}
$$

Proof. From (3.6) for $\alpha=2$ and 3 we obtain

$$
\theta_{2}=\omega_{1} \circ J_{3}, \quad \theta_{3}=-\omega_{1} \circ J_{2} .
$$

Then, according to (3.5), we get

$$
\omega_{2}=\omega_{3}=0
$$

and therefore we obtain (3.7). 
From Proposition 3.4 and Proposition 3.5 we have directly

Corollary 3.6. Let $(M, H, G)$ be an almost hypercomplex $N H$-manifold, belonging to the class $\mathcal{W}_{3}$ with respect to $J_{2}$ and $J_{3}$. If $(M, H, G)$ is quaternionic Kählerian, then it is a hyper-Kähler NH-manifold, $i$. e.

$$
(M, H, G) \in \mathcal{W}_{3}\left(J_{2}\right) \cap \mathcal{W}_{3}\left(J_{3}\right) \quad \Rightarrow \quad(M, H, G) \in \mathcal{K} .
$$

Having in mind Propositions 3.3 3.5. Corollary 3.6 and Theorem 1.1, we give the following

Conclusion 3.7. Let a quaternionic Kähler $N H$-manifold $(M, H, G)$ be in some of the classes $\mathcal{W}_{1} \oplus \mathcal{W}_{2}$ (and in particular $\mathcal{W}_{0}, \mathcal{W}_{1}$ and $\mathcal{W}_{2}$ ), $\mathcal{W}_{2} \oplus \mathcal{W}_{3}$ and $\mathcal{W}_{3}$ with respect to both of the structures $J_{2}$ and $J_{3}$. Then $(M, H, G)$ is a flat hyper-Kähler $N H$-manifold. The unique class in (1.8) with some condition for $\nabla J_{2}$ and $\nabla J_{3}$, where $(M, H, G)$ is not flat hyper-Kählerian, is $\mathcal{W}_{1} \oplus \mathcal{W}_{3}$ and their manifolds are determined by (3.7).

\section{Non-Hyper-Kähler QUATERnionic KÄHLER NH-MANifOldS}

In this section we will characterize the manifold satisfying the conditions of Proposition 3.5. It is a non-hyper-Kähler quaternionic Kähler NH-manifold.

We apply (3.8) to (2.2) and obtain the square norms of the non-zero quantities $\nabla J_{2}$ and $\nabla J_{3}$ in the considered case as follows:

$$
\left\|\nabla J_{2}\right\|^{2}=\left\|\nabla J_{3}\right\|^{2}=-4 n \omega_{1}\left(\Omega_{1}\right)
$$

where $\Omega_{1}$ is the corresponding vector to $\omega_{1}$ with respect to $g$.

Corollary 4.1. Let $(M, H, G)$ be a quaternionic Kähler NH-manifold, determined by a local 1-form $\omega_{1}$ in (3.7). It is an isotropic hyper-Kähler NH-manifold iff the corresponding vector $\Omega_{1}$ to $\omega_{1}$ with respect to $g$ is an isotropic vector regarding $g$. $\square$

Using (3.8) and Proposition 2.9, for the considered manifolds here we have

Proposition 4.2. Let $\omega_{1}$ be the local 1-form of a quaternionic Kähler NH-manifold of dimension $4 n \geq 8$, determined by (3.7). Then $\omega_{1}$ satisfies the condition

$$
\mathrm{d} \omega_{1}(x, y)=-\frac{\tau}{4 n^{2}} g_{1}(x, y) \text {. }
$$

According to (3.7) we have $F_{1}(x, y, z)=\left(\nabla_{x} g_{\alpha}\right)(y, z)=0$ and then we obtain $\mathrm{d} g_{1}(x, y, z)=\mathfrak{S}_{x, y, z}\left\{F_{1}(x, y, z)\right\}=0$. Hence and (4.1) we establish that $\tau=$ const, i. e. $(M, H, G)$ determined by (3.7) has a constant scalar curvature. Then, having in mind Theorem 2.7, we get the following

Proposition 4.3. Quaternionic Kähler NH-manifolds determined by (3.7) for dimension $4 n \geq 8$ are Ricci-symmetric, i. e. $\nabla \rho=0$.

As in Proposition 2.4 for an arbitrary quaternionic Kähler NH-manifold, in the following proposition we give a necessary and sufficient condition the considered manifold in this section be flat.

Proposition 4.4. Let $(M, H, G)$ be a quaternionic Kähler NH-manifold, determined by a non-zero local 1-form $\omega_{1}$ in (3.7). Then $(M, H, G)$ is flat non-hyperKählerian iff $\omega_{1}$ is closed. 
Proof. Since $M$ is a Kähler manifold with respect to $J_{1}$, then $R$ is Kählerian with respect to $J_{1}$.

Having in mind (3.8) and (2.4), we have that $\eta_{1}=\mathrm{d} \omega_{1}$ in the considered case. Then identity (2.8) takes the following form

$$
\begin{aligned}
R\left(x, y, J_{2} z, J_{2} w\right) & =R\left(x, y, J_{3} z, J_{3} w\right) \\
& =-R(x, y, z, w)+d \omega_{1}(x, y) g\left(J_{1} z, w\right) .
\end{aligned}
$$

It is clear that $R$ is a Kähler-type tensor with respect to $H=\left(J_{\alpha}\right)$ iff $\omega_{1}$ is closed. Hence, according to Theorem [1.2, we obtain the statement.

Corollary 4.5. Let $(M, H, G)$ be a quaternionic Kähler NH-manifold, determined by a non-zero local 1-form $\omega_{1}$ in (3.7). Then $(M, H, G)$ is flat non-hyper-Kählerian iff the following identity is valid

$$
d \theta_{\alpha}(x, y)+d \theta_{\alpha}\left(J_{1} x, J_{1} y\right)-d \theta_{\alpha}\left(J_{2} x, J_{2} y\right)-d \theta_{\alpha}\left(J_{3} x, J_{3} y\right)=0
$$

for $\alpha=2$ or $\alpha=3$.

Proof. It follows directly from Proposition 4.4 and the relations $\omega_{1}=-\theta_{2} \circ J_{3}=$ $\theta_{3} \circ J_{2}$ in (3.7).

\section{REFERENCES}

[1] D.V. Alekseevsky and S. Marchiafava, Quaternionic structures on a manifold and subordinated structures. Ann. Mat. Pura Appl. (IV) CLXXI (1996), 205-273. 1013

[2] G. Ganchev and A. Borisov, Note on the almost complex manifolds with a Norden metric. C. R. Acad. Bulgare Sci. 39 (1986), 31-34. 3

[3] A. Gray and L.M. Hervella, The sixteen classes of almost Hermitian manifolds and their linear invariants. Ann. Mat. Pura Appl. (IV) CXXIII (1980), 35-58. 3

[4] K. Gribachev and M. Manev, Almost hypercomplex pseudo-Hermitian manifolds and a 4-dimensional Lie group with such structure. J. Geom. 88, no. 1-2 (2008), 41-52. arXiv:0711.2798 10

[5] K. Gribachev, M. Manev and S. Dimiev, On the almost hypercomplex pseudo-Hermitian manifolds. In: Trends of Complex Analysis, Differential Geometry and Mathematical Physics (S. Dimiev and K. Sekigawa, eds.), pp. 51-62, World Sci. Publ., 2003. arXiv:0809.0784 1 [ 3

[6] M. Manev, Tangent bundles with Sasaki metric and almost hypercomplex pseudo-Hermitian structure. In: Topics in Almost Hermitian Geometry and Related Fields. Proc. in honor of Prof. K. Sekigawa's 60th birthday (Y. Matsushita, E. Garcia-Rio, H. Hashimoto, T. Koda and T. Oguro, eds.), pp. 170-185, World Sci. Publ., 2005. arXiv:0804.2809 3

[7] M. Manev, A connection with parallel torsion on almost hypercomplex manifolds with pseudoHermitian structure. (submitted) arXiv:0806.3831 2 3 4

[8] M. Manev and K. Sekigawa, Some four-dimensional almost hypercomplex pseudo-Hermitian manifolds. In: Contemporary Aspects of Complex Analysis, Differential Geometry and Mathematical Physics (S. Dimiev and K. Sekigawa, eds.), pp. 174-186, World Sci. Publ., 2005. arXiv:0804.2814 3

University of Plovdiv

Faculty of Mathematics and Informatics

Department of Geometry

236 Bulgaria Blvd

Plovdiv 4003

Bulgaria

e-mail: mmanev@uni-plovdiv.bg

http://fmi.uni-plovdiv.bg/manev 